\title{
Design and implementation of the system of the electric vehicle photovoltaic charging
}

\author{
LI Xiao-xia
}

(Hunan Railway Professional Technology College, Zhuzhou, 412001)

Abstract: Induction integrated starter/generator(ISG) of automobile has two function, one function is used as starter motor ,the other function is used as generator. By using this machine, the special starter can be leave out. The configuration of automobile can be simple and reasonable. The control platform of induction ISG, which is constructed of DSP control system and power electronic, is designed. The hardware and software of control platform are designed.TMS320F2812 is used as control core of the hardware. The hardware includes master control circuit, periphery circuit, master power circuit and power supply. The software includes communication software to personal computer and starter/generator double function control software. The hardware is debugged successfully. The communication software to personal computer is also debugged successfully。

The control platform has general-purpose. It can be used to control many electrical machine.

Key words: Electric vehicle; power battery; photovoltaic battery; charging device 。

\section{Introduction}

In order to further reduce fuel consumption, improve energy efficiency, more and more modern cars use a variety of electronic control auxiliary device to replace the traditional mechanical auxiliary device driver; in order to improve the vehicle's comfort and safety, the demand of modern automobile electric power is also increasing. The electrical power requirements increase, will cause the generator volume, weight and drive engine, starter its size and weight increased greatly, but the car space is limited, so the research of new automobile electrical technology is very urgent.

Starter / generator with a motor starting and generating double function auto integration, can save the special starting motor, it is convenient to choose the electric motor or generator working state, to avoid the rapid start the engine idling, increase power, automobile power system save space, reduce weight, save fuel, the ideal way to reduce carbon dioxide emissions, has become an important direction of today's new car electric technology development.

\section{System composition and principle}

The integrated starter / generator system consists of an engine, a gearbox, an 
induction motor, a power electronic converter, a controller, a battery and a load.

When the engine is started, the controller and the power electronic converter control of asynchronous motor in electric state, as the starting motor; after starting the engine, and then transferred to the control of asynchronous motor power, generate electricity supply battery and electrical load of asynchronous motor driven by the engine.

In order to obtain high performance starter / generator motor control system, must rely on the advanced control algorithm (such as space vector control and direct torque control) and realize the control algorithm of the hardware platform, the digital signal processor (Digital Signal Processor, referred to as DSP) provides a good hardware platform for the realization of control algorithm with high performance. This project is based on the TMS320F2812 as the core controller of the asynchronous start / power generation system control platform, in order to obtain high-perfo rmance automotive power system, to promote the development of automotive electrical technology.

\section{Hardware design of system hardware design 3.1 Whole design}

According to the function of the control platform and the design requirements of the control platform, the control platform consists of four parts:

1. Main control circuit. With DSP as the control core, with the clock, reset, JTAG interface, external expansion RAM, CPLD decoding circuit, D/A conversion circuit, to achieve the operation and output of the control strategy.

2. Peripheral circuit. The utility model comprises a signal detection circuit, a A/D input conditioning circuit, a driving circuit and a fault protection circuit, and realizes the detection of the signal, the driving of the power circuit and the protection of the whole system.

3. Main circuit of power electronic converter. Realization of energy transformatio $-\mathrm{n}$ and bidirectional flow.

4. The power adapter。The utility model is used for supplying power to the main control circuit and the peripheral circuit.

\subsection{Design of main unit circuit}

\subsubsection{Signal detection circuit}

In this system, 4 current (three phase current, DC bus current) and 1 voltage (DC bus voltage) are measured. The detection principle of the 4 current is the same as the A phase current detection circuit shown in Fig.2. The current is detected by LA Holzer current sensor CSM040G in Fig. 1. The output of the Holzer current sensor is converted into a voltage signal by a precision resistor $\mathrm{R} 1$ and then connected to a 
voltage follower OP-07 to improve the output impedance.

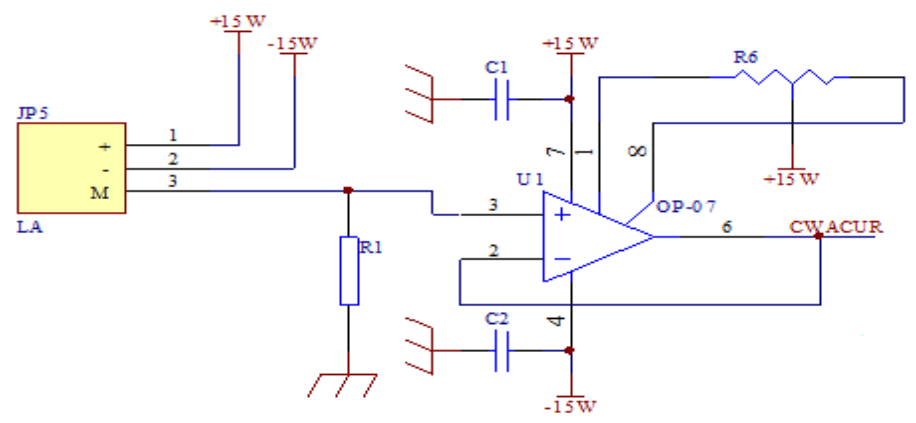

Fig.1 A phase current detection circuit

Voltage detection circuit shown in Fig.2. The voltage is detected by the LM closed loop Holzer voltage sensor VSM025A. Similarly, the output of the sensor is converted into a voltage signal by a precision resistor $\mathrm{R} 5$ and then connected to a voltage follower OP-07 to improve the output impedance.

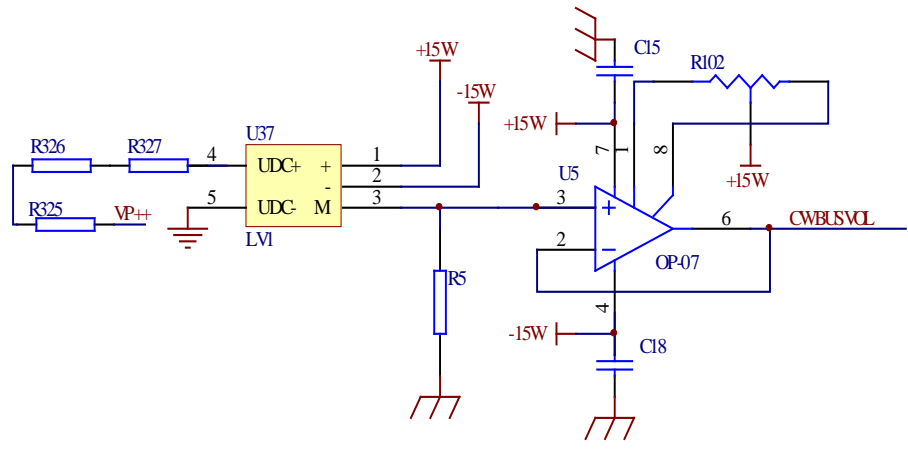

Fig. 2 voltage detection circuit

\subsubsection{A/D input conditioning circuit}

TMS320F2812 DSP internal ADC analog voltage input range of the channel is 0 $\sim 3 \mathrm{~V}$, if beyond this range will damage the DSP device, so the analog to digital conversion before must first undergo a $A / D$ input to the permissible voltage range within the conditioning circuit in the external physical quantity detection into the ADC. If the AC signal is measured, the conditioning circuit must also realize the AC to DC voltage conversion. Therefore, the reference power supply of the A/D input conditioning circuit must be designed first. A/D conditioning circuit set voltage reference power supply as shown in Fig. 3. LM336 is a precision 5V fast regulation diode, its third terminal for the reference voltage and temperature coefficient adjustment 。

A/D input conditioning circuit shown in Fig.4. By the TL084 operational amplifier and peripheral resistance capacitance elements to realize AC conditioning transformation and output DC DC amplitude is measured, the external voltage follower and reverse phase reference voltage and the amplitude of the zoom, adjust to the magnitude required, to deal with the subsequent circuit; output uses diode on the 
physical quantity after conditioning for voltage clamp a protective, low pass filter capacitor.

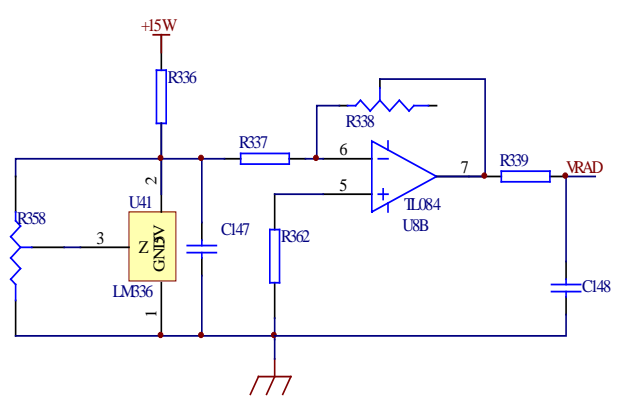

Fig.3 A/D reference power supply circuit

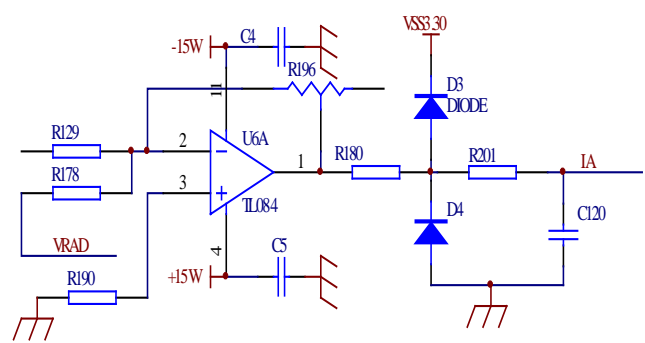

Fig.4 A/D input conditioning circuit

\subsubsection{Photoelectric isolation circuit}

The main circuit of the system operates in the high voltage and high current switching mode, and the control circuit requires the power supply voltage to be stable and without interference. Photoelectric isolation. PWM pulse isolation circuit as shown in Fig. 5. The optocoupler selects 6N137, which is a high speed optocoupler for single channel applications. The PWM switch signal by the GAL16V8 after the judgment is input into the input terminal of the optocoupler, if the light emitting diode low level optocoupler conduction, the output will optocoupler output low power module corresponding pipe disconnect switch power module for high level time coupling output high level corresponding to the open tube.

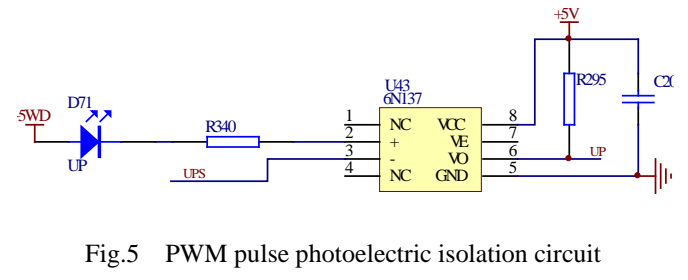

\subsubsection{Protection circuit}

The function of the protection circuit is to produce the fault protection signal, which plays the role of the protection system. The source of the system is defined according to the need. According to the actual situation can also increase the corresponding protection management, by modifying the CPLD part of the program can be 。

The protection signal of IPM is generated directly from the module, we only need to draw it out, and the actual signal value of current protection and voltage protection comes from the detection circuit of Fig. 1 and Fig. 2. Physical protection of the AC and DC two, DC signal can directly enter the comparator and the maximum setting value, and the first AC signal through a precise absolute value circuit, the AC signal into a DC signal in order to compare the comparator into. Protection threshold adjustment circuit shown in Fig. 6. 


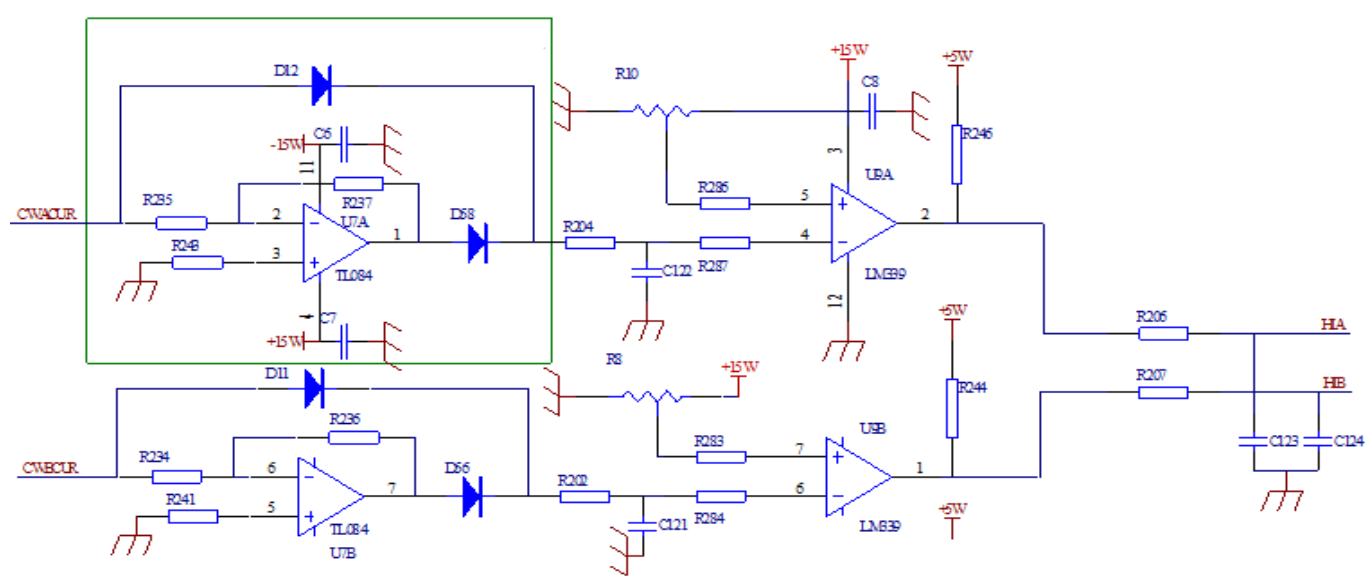

Fig. 6 protection threshold adjusting circuit

As the start / power generation control system is composed of a number of functional modules, the structure is more complex than the general MCU control system, there may be more cases of failure. In order to realize the real-time monitoring and alarm for the main faults, we need to unify the management of the fault sources, but can not connect each fault to the DSP pin.

Fault indication and alarm circuit shown in Fig.7. This system adopts 74LS30 eight input NAND gate to deal with the fault signal, regardless of failure to make alarm signals.

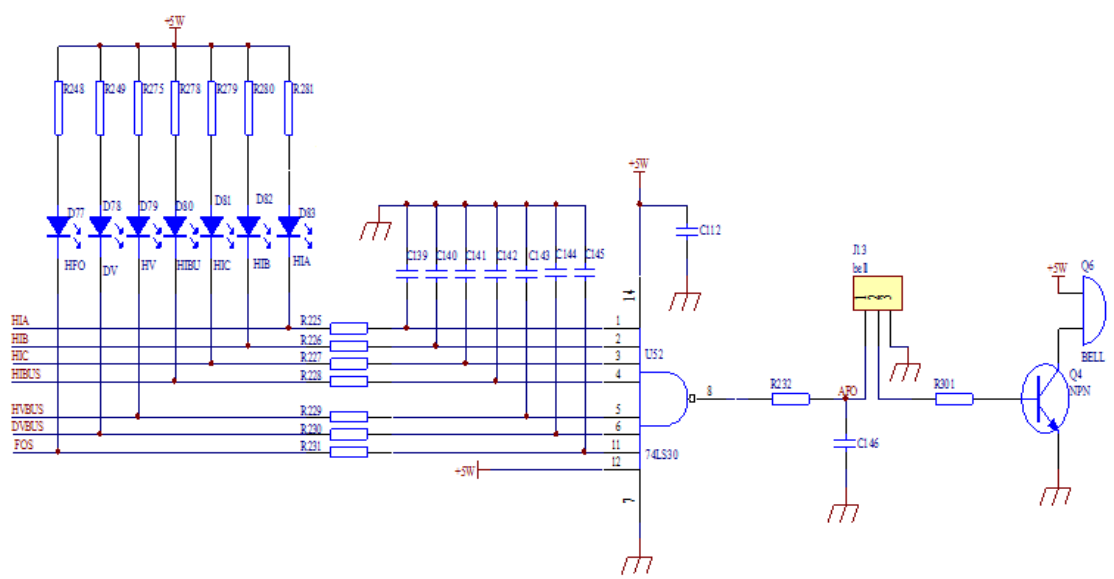

Fig. 7 fault indication and alarm circuit

\section{4 system software design}

There are many parameters to be observed in the experiment and debugging phase. Therefore, although the intelligent touch screen module is set up on the platform, the communication interface is reserved for the on-line debugging and remote monitoring of the upper computer.

PC and starter / generator system control platform using RS232 serial port for data communication, the baud rate is 9600 by default, through the platform monitoring software can launch platform, setting speed, motor speed setting the platform platform to check, check the motor current and the actual speed of the motor start and stop.

There are four buttons on the interface, which are the starting platform, setting 
frequency, exit system and custom instruction set.

The agreement can set their own instructions, from the "custom command input" input box to enter the command, you can test the command, without the need to re design the compiler platform monitoring software.

\section{Conclusion}

At present, the hardware of the control system of induction motor starter / generator system has been designed and debugged successfully. 750W asynchronous motor using VVVF control strategy in the operation of the control platform running without load. 。

The follow-up work is the control strategy of starter / generator asynchronous motor research, real-time control software, the auto Induction Starter / generator system to achieve double function, the experimental system was successfully developed, at the same time improve the touch screen man-machine interface, so that it can escape from the computer, run independently 。

\section{Acknowledgments}

This work was financially supported by Scientific research project of Hunan Provincial Department of Education (Item number: 16C1048)

\section{References:}

[1] Yin Zhan Wen, Liu Hao. Research and design of on board photovoltaic charging system for electric vehicle [J]. Journal of Henan University Of Urban Construction. 2012 21(04):57-59.

[2] Shen Wei,Wang Xiao Kan,Sun Zhonglian. Research and design of intelligent monitoring system for electric vehicle charging station [J]. East China electric power. 2011 39(06):1000-1003.

[3] Luo Zhuowei,Hu Zechun,Song Yonghua. Electric vehicle charging load calculation method [J]. Power system automation. 2011 35(14):36-40.

[4] Jiang Hao. Harmonic suppression and elimination of electric vehicle charging station [J]. Guangdong power. 2010 23(08):16-19.

[5] MEHDIE,KENT C,JASON S.Rapid-charge electric-vehicle stations[J]. IEEE Trans on Power Delivery, 2 010,25(03): 1883-1887. 